\title{
Chapter 25 \\ On the abstract density topologies generated by lower and almost lower density operators
}

\author{
JACEK HEJDUK, RENATA WIERTELAK
}

2010 Mathematics Subject Classification: 54A10, 28 A05.

Key words and phrases: lower density operator, density topology.

\subsection{Introduction}

In the real analysis we often deal with the different kind of density topologies. The most fundamental one is the classical density topology in the family of Lebesgue measurable sets introduced by Haupt and Pauc (1952, see [8]), and intensively investigated by Goffman, Waterman (1961, see [6]), Goffman, Neugebauer, Nishiura (1961, see [5]) and Tall (1976, see [24]). However, the idea of density we can found in monograph of Hobson "The theory of functions of a real variable and the theory of Fourier's series" where is considered so called metric density (see [14]). The interesting analogue of the classical density in measure turned out to be the density topology introduced in the family of Baire sets on the real line by Poreda, Wagner-Bojakowska and Wilczyński (1985, see [22]). The both density topologies introduced by the sets of density points require some properties of the operator of density points, which is a special case of the lower density operator usually defined on an abstract measurable space. Many interesting examples of density topologies introduced and investigated recently based on lower and almost lower density operators. At this moment it is worth mentioning fundamental paper of Maharam (1958, 
see [20]) and Hamlett, Janković, Rose (1993, see [7]) concerning lower density operators in the general case for measure and category. We have decided to collect as much as possible different properties of topologies generated by lower and almost lower density operators on arbitrary measurable space. The ideas of proofs are taken from [2], [18], [29], [30], where they are presented in a special case of the classical density topology and $\mathcal{I}$-density topology.

\subsection{The case of lower density operators}

Let $X$ be a nonempty set, $\mathcal{S}$ be a $\sigma$-algebra of sets from $X$ and $\mathcal{J} \subset \mathcal{S}$ be a proper $\sigma$-ideal.

Definition 25.1. We shall say that an operator $\Phi: \mathcal{S} \rightarrow \mathcal{S}$ is a lower density operator on a measurable space $(X, \mathcal{S}, \mathcal{J})$ if

(i) $\Phi(\emptyset)=\emptyset, \quad \Phi(X)=X$;

(ii) $\forall_{A, B \in \mathcal{S}} \quad \Phi(A \cap B)=\Phi(A) \cap \Phi(B)$;

(iii) $\forall_{A, B \in \mathcal{S}} \quad(A \triangle B \in \mathcal{J} \Rightarrow \Phi(A)=\Phi(B))$;

(iv) $\forall_{A \in \mathcal{S}} \quad A \triangle \Phi(A) \in \mathcal{J}$.

Definition 25.2. We shall say that a topology $\tau$ is an abstract density topology on $X$ if there exists a lower density operator on $(X, \mathcal{S}, \mathcal{J})$ such that $\tau=\mathcal{T}_{\Phi}$, where $\mathcal{T}_{\Phi}=\{A \in \mathcal{S}: A \subset \Phi(A)\}$.

Topology $\mathcal{T}_{\Phi}$ is called generated by a lower density operator on $(X, \mathcal{S}, \mathcal{J})$.

Theorem 25.3. Let $\tau$ be an abstract density topology on $X$ generated by a lower density operator $\Phi$ on $(X, \mathcal{S}, \mathcal{J})$. Then

a) $A \in \mathcal{J}$ if and only if $A$ is $\tau$-closed and $\tau$-nowhere dense;

b) if $A \in \mathcal{J}$, then $A$ is $\tau$-closed and $\tau$-discrete;

c) $\mathcal{J}=\mathcal{M}(\tau)$, where $\mathcal{M}(\tau)$ is the family of meager sets with respect to $\tau$;

d) $A \in \mathcal{S}$ if and only if $A$ is union of a $\tau$-open and a $\tau$-closed set;

e) $\operatorname{Bor}(\tau)=\mathcal{B}(\tau)=\mathcal{S}$, where $\operatorname{Bor}(\tau)$ is the family of Borel sets and $\mathcal{B}(\tau)$ is the family of Baire sets with respect to $\tau$;

f) $\langle X, \tau\rangle$ is a Baire space;

g) $\tau=\{\Phi(A) \backslash B: A \in \mathcal{S}, B \in \mathcal{J}\}$.

Moreover, if $\mathcal{J}$ contains all singletons, then

h) $A \in \mathcal{J}$ if and only if $A$ is $\tau$-closed and $\tau$-discrete;

i) $A$ is $\tau$-compact if and only if $A$ is finite; 
j) $\langle X, \tau\rangle$ is neither a first countable, nor a second countable, nor a separable space;

$k)$ if $\mathcal{J}$ contains an uncountable set, then $\langle X, \tau\rangle$ is not a Lindelöf space;

l) every sequence consisting of different terms of $X$ does not contain $\tau$ convergent subsequences.

Proof. a) If $A \in \mathcal{J}$, then $X \backslash A \in \tau$ and $\operatorname{int}_{\tau}(A)=\emptyset$. Thus $A$ is $\tau$-closed and $\tau$ nowhere dense. Conversely, if $A$ is $\tau$-closed and $\tau$-nowhere dense and $A \notin \mathcal{J}$, then $A \in \mathcal{S} \backslash \mathcal{J}$ and $\Phi(A) \cap A \in \tau \backslash\{\emptyset\}$. It contradicts the fact that $A$ is $\tau$ nowhere dense.

Conditions $b$ ) and $c$ ) are a consequence of $a$ ).

d) It is sufficient to observe that if $A \in \mathcal{S}$, then $A=(A \cap \Phi(A)) \cup(A \backslash \Phi(A))$. Obviously $A \cap \Phi(A) \in \tau$ and $A \backslash \Phi(A)$ is $\tau$-closed.

It is clear that condition $d$ ) implies $e$ ).

f) If $A \in \tau \backslash\{\emptyset\}$, then $A \notin \mathcal{J}$. By condition $c) A \notin \mathcal{M}(\tau)$. Hence $\langle X, \tau\rangle$ is a Baire space.

g) If $A \in \mathcal{S}$ and $B \in \mathcal{J}$, then $\Phi(A) \backslash B \in \tau$. Let $A \in \tau$. Then $A \subset \Phi(A)$ so $A=$ $\Phi(A) \backslash(\Phi(A) \backslash A)$. Since $\Phi(A) \backslash A \in \mathcal{J}$, we have $\tau \subset\{\Phi(A) \backslash B: A \in \mathcal{S}, B \in \mathcal{J}\}$ and the proof of $g$ ) is completed.

$h)$ In virtue of $b$ ) it is sufficient to show that if $A$ is $\tau$-closed and $\tau$-discrete, then $A \in \mathcal{J}$. Suppose that $A \neq \emptyset$. Obviously $A \in \mathcal{S}$ and for every $x \in A$ there exists a set $V_{x} \in \tau$, such that $x \in V_{x}$ and $V_{x} \cap A=\{x\}$. Thus

$$
x \in V_{x} \subset \Phi\left(V_{x}\right)=\Phi\left(V_{x} \backslash\{x\}\right) \subset \Phi(X \backslash A) .
$$

Hence $A \subset \Phi(X \backslash A)$. It follows that $A=\Phi(X \backslash A) \backslash(X \backslash A) \in \mathcal{J}$.

i) Assume that a set $A$ is compact and infinite. Let $B \subset A$ be a countable infinite set. Then the family $\{(X \backslash B) \cup\{x\}\}_{x \in B}$ is a $\tau$-open cover of $A$ without a finite subcover. The converse implication in condition $i$ ) is obvious.

j) Let $x \in X$ and $\left\{E_{n}\right\}_{n \in \mathbb{N}}$ be a sequence of $\tau$-open sets containing $x$. Let $x_{n} \in E_{n} \backslash\{x\}$ for every $n \in \mathbb{N}$. Putting $E=E_{1} \backslash\left\{x_{n}: n \in \mathbb{N}\right\}$, we have $E \in \tau$, $x \in E$ and $E$ does not contain set $E_{n}$ for $n \in \mathbb{N}$. So the first countability axiom is not satisfied. Also $\langle X, \tau\rangle$ is not a second countable space.

At the same time $\langle X, \tau\rangle$ is not a separable space. Indeed, taking into account a countable and dense set $A \subset X$ we have $A \in \mathcal{J}$ and therefore $X=\operatorname{cl}_{\tau}(A)=$ $A \in \mathcal{J}$, so $X \in \mathcal{J}$. It contradicts the fact that $\mathcal{J}$ is a proper $\sigma$-ideal.

$k)$ Let $D \in \mathcal{J}$ be an uncountable set. Then the family $\{(X \backslash D) \cup\{x\}\}_{x \in D}$ is a $\tau$-open cover of $X$ without a countable subcover. So that $\langle X, \tau\rangle$ is not a Lindelöf space. 
l) If $\left\{x_{n}\right\}_{n \in \mathbb{N}}$ is a sequence consisting of different terms of $X$, then by $h$ ) for every subsequence $\left\{x_{n_{k}}\right\}_{k \in \mathbb{N}}$ and $x \in X$ we get that $x \in\left(X \backslash \bigcup_{k=1}^{\infty}\left\{x_{n_{k}}\right\}\right) \cup\{x\} \in$ $\tau$. It means that there is no $\tau$-convergent subsequence of $\left\{x_{n}\right\}_{n \in \mathbb{N}}$.

Theorem 25.4. Let $\tau$ be a topology on $X$. Then the following conditions are equivalent:

(i) $\tau$ is an abstract density topology;

(ii) $\tau$ has the following properties:

(a) $A \in \mathcal{J}$ if and only if $A$ is $\tau$-closed and $\tau$-nowhere dense;

(b) $\mathcal{B}(\tau)=\mathcal{S}$.

Proof. Implication $(i) \Rightarrow(i i)$ follows from the previous theorem.

(ii) $\Rightarrow(i)$ Notice that $\langle X, \tau\rangle$ is a Baire space. Let $A \in \mathcal{S}=\mathcal{B}(\tau)$. By Theorem 4.6 from [21] the set $A$ has the unique representation in the form $G \triangle P$, where $G$ is regular $\tau$-open (i.e. $G=\operatorname{int}_{\tau}\left(\operatorname{cl}_{\tau}(G)\right)$ ) and $P \in \mathcal{J}$. Put $\Phi(A)=G$.

Obviously $\Phi(\emptyset)=\emptyset, \Phi(X)=X$, and $A \triangle \Phi(A) \in \mathcal{J}$ for $A \in \mathcal{S}$. Moreover, if $A \triangle B \in \mathcal{J}$, then $\Phi(A)=\Phi(B)$.

Now let $A, B \in \mathcal{S}$ and $A=G_{1} \triangle P_{1}, B=G_{2} \triangle P_{2}$, where $G_{1}, G_{2}$ are regular $\tau$-open and $P_{1}, P_{2} \in \mathcal{J}$. There exists $P_{3} \in \mathcal{J}$ such that $A \cap B=\left(G_{1} \cap G_{2}\right) \triangle P_{3}$. It follows that

$$
\Phi(A) \cap \Phi(B)=G_{1} \cap G_{2}=\Phi\left(G_{1} \cap G_{2}\right)=\Phi(A \cap B) .
$$

Therefore $\Phi$ is a lower density operator on $(X, \mathcal{S}, \mathcal{J})$. We shall prove that $\mathcal{T}_{\Phi}=$ $\tau$. If $A$ is $\tau$-open, then by Theorem 4.5 from [21] we have $A=G \backslash P$, where $G$ is regular $\tau$-open and $P$ is $\tau$-closed and $\tau$-nowhere dense. Thus $A \in \mathcal{S}$ and $A \subset G=\Phi(A)$. Hence $\tau \subset \mathcal{T}_{\Phi}$. Suppose now that $A \in \mathcal{T}_{\Phi}$, so $A \in \mathcal{S}, A \subset \Phi(A)$ and $A \triangle \Phi(A) \in \mathcal{J}$. It follows that $A=\Phi(A) \backslash P$, where $P \in \mathcal{J}$. Since $P$ is $\tau$-closed, $A$ is $\tau$-open.

Corollary 25.5. If $\mathcal{J}$ contains all singletons, then the operator $\Phi$ described in the proof of the above theorem has the following form:

$$
\forall_{A \in \mathcal{S}} \quad \Phi(A)=\operatorname{int}_{\tau}\left\{x \in X: x \in \operatorname{int}_{\tau}(A \cup\{x\})\right\} .
$$

Proof. Let $A \in \mathcal{S}$ and $\Phi_{1}(A)=\operatorname{int}_{\tau}\left\{x \in X: x \in \operatorname{int}_{\tau}(A \cup\{x\})\right\}$. First we show that if $A, B \in \mathcal{S}$ and $A \triangle B \in \mathcal{J}$, then $\Phi_{1}(A)=\Phi_{1}(B)$. It is clear that $A=B \triangle C$, where $C \in \mathcal{J}$. We demonstrate that $\Phi_{1}(A) \subset \Phi_{1}(B)$. Suppose that $x \in \Phi_{1}(A)$. Hence $x \in \Phi_{1}(B \triangle C)$ and $x \in \operatorname{int}_{\tau}((B \triangle C) \cup\{x\})$. There exists a $\tau$-open set $W_{x} \ni x$ such that $W_{x} \subset((B \triangle C) \cup\{x\})$. Therefore $W_{x} \backslash(C \backslash\{x\}) \subset(B \cup\{x\})$ 
and $x \in W_{x} \backslash(C \backslash\{x\})$ which is a $\tau$-open set. It follows that $x \in \operatorname{int}_{\tau}(B \cup\{x\})$ and $\Phi_{1}(A) \subset \Phi_{1}(B)$. Similarly we can show that $\Phi_{1}(B) \subset \Phi_{1}(A)$.

Now let $A=V \triangle P$, where $V$ is regular $\tau$-open and $P \in \mathcal{J}$. Then $\Phi(A)=V$ and $\Phi_{1}(A)=\Phi_{1}(V)$. It is sufficient to prove that $V=\Phi_{1}(V)$. Since $V \subset \Phi_{1}(V)$ it remains to show that $\Phi_{1}(V) \subset V$. Suppose that $x \in \Phi_{1}(V)$ and $x \in W \in \tau$. Since $x \in \operatorname{int}_{\tau}(V \cup\{x\})$, there exists a set $W_{x} \in \tau$ such that $x \in W_{x} \subset V \cup\{x\}$. It is clear that $x \in\left(W \cap W_{x}\right) \neq \emptyset$. Moreover, $\left(W \cap W_{x}\right) \backslash\{x\} \neq \emptyset$, because $\mathcal{J}$ contains all singletons and $\left(W \cap W_{x}\right) \subset \Phi\left(W \cap W_{x}\right)$. Consequently $\left(W \cap W_{x}\right) \backslash$ $\{x\} \subset V$ and $W \cap V \neq \emptyset$. It implies that the set $\left\{x \in X: x \in \operatorname{int}_{\tau}(V \cup\{x\})\right\}$ is $\tau$-open. Therefore $\Phi_{1}(V)=\operatorname{int}_{\tau} \Phi_{1}(V) \subset \operatorname{int}_{\tau}\left(c l_{\tau} V\right)=V$.

Theorem 25.6. Let $\Phi$ be a lower density operator on $(X, \mathcal{S}, \mathcal{J})$. Then the family

$$
\mathcal{T}_{\Phi}=\{A \in \mathcal{S}: A \subset \Phi(A)\}
$$

is a topology on $X$ if and only if the pair $(\mathcal{S}, \mathcal{J})$ has the hull property.

Proof. Sufficiency. Let $\Phi$ be a lower density operator on $(X, \mathcal{S}, \mathcal{J})$. Obviously $\emptyset, X \in \mathcal{T}_{\Phi}$ and the family $\mathcal{T}_{\Phi}$ is closed under finite intersections. Let $\left\{A_{t}\right\}_{t \in T} \subset$ $\mathcal{T}_{\Phi}$. We shall prove that $\bigcup_{t \in T} A_{t} \in \mathcal{T}_{\Phi}$. Let $B$ be a $\mathcal{S}$-measurable kernel of the set $\bigcup_{t \in T} A_{t}$. Hence for every $t \in T$ we have $\left(A_{t} \cap B\right) \triangle A_{t} \in \mathcal{J}$ and

$$
B \subset \bigcup_{t \in T} A_{t} \subset \bigcup_{t \in T} \Phi\left(A_{t}\right)=\bigcup_{t \in T} \Phi\left(A_{t} \cap B\right) \subset \Phi(B) .
$$

Since $\Phi(B) \backslash B \in \mathcal{J}$, we have $\bigcup_{t \in T} A_{t} \in \mathcal{S}$. Moreover, it is obvious that $\bigcup_{t \in T} A_{t} \subset \Phi\left(\bigcup_{t \in T} A_{t}\right)$. Therefore $\bigcup_{t \in T} A_{t} \in \mathcal{T}_{\Phi}$.

Necessity. Obviously $\mathcal{T}_{\Phi}$ is an abstract density topology on $(X, \mathcal{S}, \mathcal{J})$. By Theorem 25.3 we get $\mathcal{B}\left(\mathcal{T}_{\Phi}\right)=\mathcal{S}$ and $\mathcal{M}\left(\mathcal{T}_{\Phi}\right)=\mathcal{J}$. The well known fact that the pair $\left(\mathcal{B}\left(\mathcal{T}_{\Phi}\right), \mathcal{M}\left(\mathcal{T}_{\Phi}\right)\right)$ has the hull property (see [17]) completes the proof.

Corollary 25.7. If $\langle X, \tau\rangle$ is a topological space such that $X \notin \mathcal{M}(\tau)$, then every lower density operator $\Phi$ on $(X, \mathcal{B}(\tau), \mathcal{M}(\tau))$ generates $\mathcal{T}_{\Phi}$ topology on $X$.

Proof. Since the pair $(\mathcal{B}(\tau), \mathcal{M}(\tau))$ has the hull property, then by Theorem 25.6 any lower density operator $\Phi$ on $(X, \mathcal{B}(\tau), \mathcal{M}(\tau))$ generates topology on $X$.

Remark 25.8. If $\Phi(A)=A$ for every set $A \in \mathcal{B}$ or, then $\Phi$ is the lower density operator on $(\mathbb{R}, \mathcal{B}$ or, $\mathcal{J})$, where $\mathcal{J}=\{\emptyset\}$, but it is clear that $\mathcal{T}_{\Phi}$ is not a topology on $\mathbb{R}$. The reason is that the pair $(\mathcal{B} o r, \mathcal{J})$ does not have the hull property . 
By the idea implemented in Theorem 25.6 we get the following topologies on the real line:

- density topology (O. Haupt, C. Pauc (1952), see [8]);

- $\mathcal{I}$-density topology (W. Poreda, E. Wagner-Bojakowska, W. Wilczyński (1985), see [22]);

- topology involving measure and category (W. Wojdowski (1989), see [33]);

- $\langle s\rangle$-density topology with respect to category (J. Hejduk, G. Horbaczewska (2003), see [12], [15]);

- $\langle s\rangle$-density topology with respect to measure (M. Filipczak, J. Hejduk (2004), see [4]);

- density topology related to category with respect to a sequence tending to zero (R. Wiertelak (2006), see [28]);

- $\langle s\rangle$-simple density topology with respect to category (V. Aversa, W. Wilczyński (2004), see [1]);

- $\Psi_{\mathcal{I}}$-density topology (E. Łazarow, A. Vizvary (2010), see [19]);

- category $\psi$-density topology (W. Wilczyński, W. Wojdowski (2011), see [31]).

Abstract density topologies allow us to find a clear and useful representation of the interior of any set. Namely, we have the following theorem which proof for the case of density topology can be found in [30].

Theorem 25.9. If $\tau$ is an abstract density topology on $X$ generated by a lower density operator $\Phi$ on $(X, \mathcal{S}, \mathcal{J})$, then for any set $A \subset X$ we have int $\tau A=$ $A \cap \Phi(B)$, where $B$ is an $\mathcal{S}$-measurable kernel of $A$.

Proof. Let $A \subset X$. By Theorem 25.6 the pair $(\mathcal{S}, \mathcal{J})$ possesses the hull property. Let $B \subset A$ be an $\mathcal{S}$-measurable kernel of $A$. Observe that $A \cap \Phi(B) \in \tau$. Indeed,

$$
A \cap \Phi(B)=(B \cap \Phi(B)) \cup((A \backslash B) \cap \Phi(B)) .
$$

Since $\Phi(B) \cap(A \backslash B) \subset(\Phi(B) \backslash B) \in \mathcal{J}$, we have $A \cap \Phi(B) \in \mathcal{S}$. Moreover, $\Phi(A \cap \Phi(B))=\Phi(B \cap \Phi(B))=\Phi(B)$. It follows that $A \cap \Phi(B) \in \tau$. Let us assume that $V \in \tau$ and $V \subset A$. We show that $V \subset A \cap \Phi(B)$. Since $V=(V \cap$ $B) \cup(V \cap(A \backslash B))$ and $V \cap(A \backslash B) \subset V \backslash B \subset A \backslash B$, we have $V \backslash B \in \mathcal{J}$ and also $V \cap(A \backslash B) \in \mathcal{J}$. Therefore $\Phi(V)=\Phi(V \cap B)=\Phi(V) \cap \Phi(B)$ which implies that $V \subset \Phi(V) \subset \Phi(B)$. Thus $V \subset A \cap \Phi(B)$.

Corollary 25.10. If $\tau$ is an abstract density topology on $X$ generated by a lower density operator $\Phi$ on $(X, \mathcal{S}, \mathcal{J})$, then $\Phi(A) \subset c_{\tau}(A)$ for every $A \in \mathcal{S}$. 
Proof. Let $A \in \mathcal{S}$. Then

$$
\operatorname{cl}_{\tau}(A)=X \backslash \operatorname{int}_{\tau}(X \backslash A)=X \backslash[(X \backslash A) \cap \Phi(X \backslash A)]=A \cup(X \backslash \Phi(X \backslash A)) .
$$

From $\Phi(A) \subset X \backslash \Phi(X \backslash A)$, it follows that $\Phi(A) \subset \operatorname{cl}_{\tau}(A)$.

Theorem 25.11. If $\tau$ is an abstract density topology on $X$ generated by a lower density operator $\Phi$ on $(X, \mathcal{S}, \mathcal{J})$, then $A \subset X$ is a regular $\tau$-open set if and only if $A=\Phi(A)$.

Proof. Necessity. Let $A$ be a regular $\tau$-open set, i.e. $A=\operatorname{int}_{\tau}\left(c l_{\tau}(A)\right)$. Then, by Theorem 25.9, $A=c l_{\tau}(A) \cap \Phi\left(c l_{\tau}(A)\right)$. In virtue of Corollary 25.10, $\Phi(A) \subset$ $c l_{\tau}(A)$. Moreover, $\Phi(A) \subset \Phi\left(c l_{\tau}(A)\right)$. It follows that $\Phi(A) \subset A$. Evidently $A \in \tau$, so that $A \subset \Phi(A)$ and finally $A=\Phi(A)$.

Sufficiency. Let $A=\Phi(A)$. Then $A \in \tau$ and $c l_{\tau}(A) \backslash A \in \mathcal{J}$. Hence

$$
\operatorname{int}_{\tau}\left(c l_{\tau}(A)\right)=c l_{\tau}(A) \cap \Phi\left(\operatorname{cl}_{\tau}(A)\right)=\operatorname{cl}_{\tau}(A) \cap \Phi(A)=\Phi(A)=A .
$$

Therefore $A$ is a $\tau$-regular open set.

Theorem 25.12. Let $\Phi_{1}, \Phi_{2}$ be the lower density operators on $(X, \mathcal{S}, \mathcal{J})$ generating $\mathcal{T}_{\Phi_{1}}, \mathcal{T}_{\Phi_{2}}$ topologies, respectively. Then $\mathcal{T}_{\Phi_{1}}=\mathcal{T}_{\Phi_{2}}$ if and only if $\Phi_{1}=\Phi_{2}$.

Proof. Sufficiency is obvious.

Necessity. Let $A \in \mathcal{S}$. Then $\Phi_{1}(A) \in \mathcal{T}_{\Phi_{1}}=\mathcal{T}_{\Phi_{2}}$. It follows that $\Phi_{2}\left(\Phi_{1}(A)\right) \supset$ $\Phi_{1}(A)$. Since $\Phi_{1}(A) \triangle A \in \mathcal{J}$, we obtain $\Phi_{2}\left(\Phi_{1}(A)\right)=\Phi_{2}(A)$ and consequently $\Phi_{2}(A) \supset \Phi_{1}(A)$. Similarly we show that $\Phi_{1}(A) \supset \Phi_{2}(A)$.

Corollary 25.13. If $\tau$ is an abstract density topology on $X$, then there exists a unique lower density operator $\Phi$ on $(X, \mathcal{S}, \mathcal{J})$ such that $\mathcal{T}_{\Phi}=\tau$.

Definition 25.14. We shall say that operators $\Phi_{1}, \Phi_{2}: \mathcal{S} \rightarrow 2^{X}$ are equivalent on $(X, \mathcal{S}, \mathcal{J})$ if $\Phi_{1}(A) \triangle \Phi_{2}(A) \in \mathcal{J}$ for every set $A \in \mathcal{S}$. It will be denoted by $\Phi_{1} \approx \Phi_{2}$.

It is evident that lower density operators $\Phi_{1}, \Phi_{2}$ on $(X, \mathcal{S}, \mathcal{J})$ are equivalent. By Theorem 25.3 and Theorem 25.12 we have the following property.

Property 25.15. If $\Phi_{1}, \Phi_{2}$ are lower density operators on $(X, \mathcal{S}, \mathcal{J})$ and $\Phi_{1}$ generates topology $\mathcal{T}_{\Phi_{1}}$ on $X$, then $\Phi_{2}$ generates topology $\mathcal{T}_{\Phi_{2}}$ on $X$ such that $\mathcal{B}\left(\mathcal{T}_{\Phi_{1}}\right)=\mathcal{B}\left(\mathcal{T}_{\Phi_{2}}\right)=\mathcal{S}$ and $\mathcal{M}\left(\mathcal{T}_{\Phi_{1}}\right)=\mathcal{M}\left(\mathcal{T}_{\Phi_{2}}\right)=\mathcal{J}$ 
Let us assume that $\langle X, \tau\rangle$ is an arbitrary topological space and $\mathcal{T}_{\Phi}$ is a topology generated by a lower density operator $\Phi$ on $(X, \mathcal{S}, \mathcal{J})$ such that $\tau \subset \mathcal{T}_{\Phi}$. In real analysis two kinds of continuity are considered: topological and restrictive.

Definition 25.16. We shall say that $f:\left\langle X, \mathcal{T}_{\Phi}\right\rangle \rightarrow\left\langle\mathbb{R}, \mathcal{T}_{\text {nat }}\right\rangle$ is topologically continuous at $x_{0} \in X$ if

$$
\underset{\varepsilon>0}{\forall} \underset{V \in \mathcal{T}_{\Phi}, x_{0} \in V}{\exists} \quad V \subset\left\{x \in X:\left|f(x)-f\left(x_{0}\right)\right|<\varepsilon\right\} .
$$

Definition 25.17. We shall say that $f:\left\langle X, \mathcal{T}_{\Phi}\right\rangle \rightarrow\left\langle\mathbb{R}, \mathcal{T}_{\text {nat }}\right\rangle$ is restrictively continuous at $x_{0} \in X$ if there exists a set $A \in \mathcal{S}$ such that $x_{0} \in \Phi(A) \cap A$ and $f_{\mid A}$ is $\tau$-continuous at $x_{0}$.

Property 25.18. If $f:\left\langle X, \mathcal{T}_{\Phi}\right\rangle \rightarrow\left\langle\mathbb{R}, \mathcal{T}_{\text {nat }}\right\rangle$ is restrictively continuous at $x_{0} \in X$, then $f$ is topologically continuous at $x_{0}$.

Proof. Let $x_{0} \in X$ and $A \in \mathcal{S}$ be a set such that $x_{0} \in \Phi(A)$ and $f_{\mid A}$ is $\tau$-continuous at $x_{0}$. Fix $\varepsilon>0$. There exists a set $V \in \tau$ such that $x_{0} \in A \cap V$ and $A \cap V \subset\left\{x \in X:\left|f(x)-f\left(x_{0}\right)\right|<\varepsilon\right\}$. Putting $W=A \cap \Phi(A) \cap V$ we obtain $W \in \mathcal{T}_{\Phi}, x_{0} \in W$ and $W \subset\left\{x \in X:\left|f(x)-f\left(x_{0}\right)\right|<\varepsilon\right\}$.

It is worth mentioning that in the case of real line there exists characterization of equivalence of restrictive and topological continuity.

Theorem 25.19 (cf. [16]). Let $\mathcal{T}_{\Phi}$ be a topology generated by a lower density operator $\Phi$ on $(\mathbb{R}, \mathcal{S}, \mathcal{J})$ such that $\mathcal{T}_{\text {nat }} \subset \mathcal{T}_{\Phi}, f:\left\langle\mathbb{R}, \mathcal{T}_{\Phi}\right\rangle \rightarrow\left\langle\mathbb{R}, \mathcal{T}_{\text {nat }}\right\rangle$ and $x_{0} \in \mathbb{R}$. Then the following conditions are equivalent:

a) $f$ is $\mathcal{T}_{\Phi}$-topologically continuous at $x_{0}$ if and only if it is restrictively continuous at $x_{0}$;

b) for every descending sequence $\left\{E_{n}\right\}_{n \in \mathbb{N}} \subset \mathcal{S}$ such that $x_{0} \in \bigcap_{n=1}^{\infty} \Phi\left(E_{n}\right)$ there exists a sequence $\left\{r_{n}\right\}_{n \in \mathbb{N}} \subset \mathbb{R}_{+}, r_{n} \searrow 0$ such that

$$
x_{0} \in \Phi\left(\bigcup_{n=1}^{\infty}\left(E_{n} \backslash\left(x_{0}-r_{n}, x_{0}+r_{n}\right)\right)\right) .
$$

Remark 25.20 (cf. [29]). In the topological space $\left\langle\mathbb{R}, \mathcal{T}_{\mathcal{I}}\right\rangle$, where $\mathcal{T}_{\mathcal{I}}$ is $\mathcal{I}$-density topology, the topological and restrictive continuity are not equivalent.

Theorem 25.21. Let $\langle X, \tau\rangle$ be a topological space and $\mathcal{T}_{\Phi}$ be a topology generated by a lower density operator $\Phi$ on $(X, \mathcal{B}(\tau), \mathcal{M}(\tau))$ such that $\tau \subset \mathcal{T}_{\Phi}$. For every function $f: X \rightarrow \mathbb{R}$ the following conditions are equivalent: 
a) $f$ has the Baire property with respect to $\tau$;

b) there exists a set $A \in \mathcal{M}(\tau)$ such that for every $x \in X \backslash A$ function $f$ is $\mathcal{T}_{\Phi}$-restrictively continuous at $x$

c) there exists a set $A \in \mathcal{M}(\tau)$ such that for every $x \in X \backslash A$ function $f$ is $\mathcal{T}_{\Phi}$-topologically continuous at $x$.

Proof. $a) \Rightarrow b$ ) Let $f$ be a function with the Baire property. There exists a set $A \in \mathcal{M}(\tau)$ such that $f_{\mid X \backslash A}$ is $\tau$-continuous. Then for every $x \in X \backslash A$ we have $x \in \Phi(X \backslash A)=X$ and $f$ is restrictively continuous at $x$.

By Property 25.18 implication $b) \Rightarrow c$ ) holds.

$c) \Rightarrow a)$ We show that $E=f^{-1}((a, b)) \in \mathcal{B}(\tau)$ for every $a, b \in \mathbb{R}$, such that $a<b$. Let $C$ be the set of $\mathcal{T}_{\Phi}$-topological continuity points of $f$. Obviously $E=(E \cap C) \cup(E \backslash C)$ and $E \backslash C \in \mathcal{M}(\tau)$. Let $z \in E \cap C$ and $\varepsilon>0$ be such that $\varepsilon<\min \{b-f(z), f(z)-a\}$. Then there exists a set $V_{z} \in \mathcal{T}_{\Phi}$ such that $z \in V_{z} \subset$ $\{x \in X:|f(x)-f(z)|<\varepsilon\}$. Putting $V_{z}^{\prime}=V_{z} \cap C$ we obtain $z \in V_{z}^{\prime}$ and $V_{z}^{\prime} \in \mathcal{T}_{\Phi}$. Hence $E \cap C=\bigcup_{z \in E \cap C} V_{z}^{\prime} \in \mathcal{T}_{\Phi} \subset \mathcal{B}(\tau)$. Therefore $E=f^{-1}((a, b)) \in \mathcal{B}(\tau)$.

\subsection{The case of almost lower density operators}

Let $(X, \mathcal{S}, \mathcal{J})$ be a measurable space, where $X$ is a nonempty set, $\mathcal{S}$ be a $\sigma$-algebra of subsets of $X$ and $\mathcal{J} \subset \mathcal{S}$ a proper $\sigma$-ideal.

Definition 25.22. We shall say that an operator $\Phi: \mathcal{S} \rightarrow 2^{X}$ is an almost lower density operator on measurable space $(X, \mathcal{S}, \mathcal{J})$ if

(i) $\Phi(\emptyset)=\emptyset, \quad \Phi(X)=X$;

(ii) $\forall_{A, B \in \mathcal{S}} \quad \Phi(A \cap B)=\Phi(A) \cap \Phi(B)$;

(iii) $\forall_{A, B \in \mathcal{S}} \quad A \triangle B \in \mathcal{J} \Rightarrow \Phi(A)=\Phi(B)$;

(iv) $\forall_{A \in \mathcal{S}} \quad \Phi(A) \backslash A \in \mathcal{J}$.

It is worthwhile noting that in the above definition instead of a $\sigma$-algebra $\mathcal{S}$ we can consider a family $\mathcal{S}$ closed under finite intersections such that $\emptyset \in \mathcal{S}$, $X \in \mathcal{S}$ and $\mathcal{J} \subset \mathcal{S}$.

The next theorem follows by the same method as in proof of sufficient condition of Theorem 25.6.

Theorem 25.23. Let $\Phi$ be an almost lower density operator on $(X, \mathcal{S}, \mathcal{J})$. If the pair $(\mathcal{S}, \mathcal{J})$ has the hull property, then the family

$$
\mathcal{T}_{\Phi}=\{A \in \mathcal{S}: A \subset \Phi(A)\}
$$

is a topology on $X$. 
We will say that $\mathcal{T}_{\Phi}$ is a topology generated by the almost lower density operator $\Phi$ on $(X, \mathcal{S}, \mathcal{J})$.

The following example shows that the inverse of the last theorem does not hold.

Example 25.24. Let $(\mathbb{R}, \mathcal{B}$ or, $\mathcal{J})$ be a measurable space, where $\mathcal{J}$ denotes the $\sigma$-ideal of countable sets. Putting

$$
\forall_{A \in \mathcal{S}} \quad \Phi(A)=\left\{\begin{array}{cl}
\mathbb{R}, & \text { if } \mathbb{R} \backslash A \in \mathcal{J}, \\
\emptyset, & \text { if } \mathbb{R} \backslash A \notin \mathcal{J},
\end{array}\right.
$$

we obtain the topology

$$
\mathcal{T}_{\Phi}=\{A \in \mathcal{B} \text { or }: A=\emptyset \vee \mathbb{R} \backslash A \in \mathcal{J}\}
$$

but the pair $(\mathcal{B}$ or, $\mathcal{J})$ does not possesses the hull property.

Example 25.25 . Let $\Phi_{1}, \Phi_{2}$ be the almost lower density operators on $(\mathbb{R}, \mathcal{L}, \mathcal{N})$ defined in the following way

$$
\begin{aligned}
& \forall_{A \in \mathcal{L}} \quad \Phi_{1}(A)= \begin{cases}\mathbb{R}, & \text { if } \mathbb{R} \backslash A \in \mathcal{N}, \\
\emptyset, & \text { if } \mathbb{R} \backslash A \notin \mathcal{N},\end{cases} \\
& \forall_{A \in \mathcal{L}} \quad \Phi_{2}(A)=\left\{\begin{array}{cc}
\mathbb{R}, & \text { if } \mathbb{R} \backslash A \in \mathcal{N}, \\
\Phi_{d}(A) \cap B, & \text { if } \mathbb{R} \backslash A \notin \mathcal{N},
\end{array}\right.
\end{aligned}
$$

where $B$ is a Bernstein set. Then $\Phi_{1} \neq \Phi_{2}$ but

$$
\mathcal{T}_{\Phi_{1}}=\mathcal{T}_{\Phi_{2}}=\{A \in \mathcal{L}: A=\emptyset \vee \mathbb{R} \backslash A \in \mathcal{N}\} .
$$

It means that the analogue of Theorem 25.12 in the case of almost lower density operators is not true. It turns out that $\Phi_{1}$ and $\Phi_{2}$ are not equivalent. However, we have the following property.

Property 25.26. Let $\Phi_{1}, \Phi_{2}$ be the almost lower density operators on $(X, \mathcal{S}, \mathcal{J})$. Then the equality $\mathcal{T}_{\Phi_{1}}=\mathcal{T}_{\Phi_{2}}$ implies that $\Phi_{1}(A) \Delta \Phi_{2}(A) \in \mathcal{J}$ for every $A \in \mathcal{T}_{\Phi_{1}}$.

Proof. Let $A \in \mathcal{T}_{\Phi_{1}}=\mathcal{T}_{\Phi_{2}}$. Then $\Phi_{1}(A)=A \cup\left(\Phi_{1}(A) \backslash A\right)$ and $\Phi_{2}(A)=A \cup$ $\left(\Phi_{2}(A) \backslash A\right)$. Hence $\Phi_{1}(A) \triangle \Phi_{2}(A) \in \mathcal{J}$ for every $A \in \mathcal{T}_{\Phi_{1}}$.

Below there are examples of topologies generated by the almost lower density operators on the real line:

- $\psi$-density topology (M. Terepeta, E. Wagner-Bojakowska, (1999), see [25]); 
- density topology with respect to the O'Malley points (W. Poreda, W. Wilczyński (2001), see [23]);

- density topology with respect to measure and category (J. Hejduk (2002), see [10]);

- complete density topology (W. Wilczyński, W. Wojdowski (2007), see [32]);

- $f$-density topology (M. Filipczak, T. Filipczak, (2008), see [3]);

- $f$-symmetrical density topology (J. Hejduk (2008), see [9]);

- simple density topology (V. Aversa, W. Wilczyński (2004), see [1]);

- density topology in the aspect of measure with respect to a sequence tending to zero (J. Hejduk, R. Wiertelak (2012), see [13]).

The same ideas as the ones used in proofs of Theorem 25.3 and Theorem 25.9 allow us to prove the next theorem.

Theorem 25.27. If $\Phi$ is an almost lower density operator on $(X, \mathcal{S}, \mathcal{J})$ generating topology $\mathcal{T}_{\Phi}$, then the following conditions are satisfied:

a) if $A \in \mathcal{J}$, then $A$ is $\mathcal{T}_{\Phi}$-closed and $\mathcal{T}_{\Phi}$-nowhere dense;

b) if $A \in \mathcal{J}$, then $A$ is $\mathcal{T}_{\Phi}$-closed and $\mathcal{T}_{\Phi}$-discrete;

c) $\mathcal{J} \subset \mathcal{M}\left(\mathcal{T}_{\Phi}\right)$;

d) if $\mathcal{J}=\mathcal{M}\left(\mathcal{T}_{\Phi}\right)$, then $\mathcal{B}\left(\mathcal{T}_{\Phi}\right) \subset \mathcal{S}$;

e) $\operatorname{int}_{\mathcal{T}_{\Phi}}(A) \subset A \cap \Phi(A)$ for every $A \in \mathcal{S}$.

Moreover, if $\mathcal{J}$ contains all singletons, then

f) $A \in \mathcal{J}$ if and only if $A$ is $\mathcal{T}_{\Phi}$-closed and $\mathcal{T}_{\Phi}$-discrete;

g) $A$ is $\mathcal{T}_{\Phi}$-compact if and only if $A$ is finite;

h) $\left\langle X, \mathcal{T}_{\Phi}\right\rangle$ is neither a first countable, nor a second countable, nor a separable space;

i) if $\mathcal{J}$ contains an uncountable set, then $\left\langle X, \mathcal{T}_{\Phi}\right\rangle$ is not a Lindelöf space;

j) every sequence consisting of different terms of $X$ does not contain $\mathcal{T}_{\Phi}$-convergent subsequence.

Remark 25.28. Topology $\mathcal{T}_{\Psi}$ obtained by the almost lower density operator

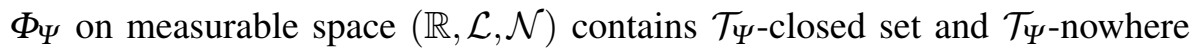
dense set from $\mathcal{L} \backslash \mathcal{N}$ (see [25]). Hence the inverse properties to $a$ ) and the equality in $c$ ) do not hold. Moreover, $\mathcal{M}\left(\mathcal{T}_{\Psi}\right)=2^{\mathbb{R}}$ (see [26]). Therefore $\left\langle\mathbb{R}, \mathcal{T}_{\Psi}\right\rangle$ is not a Baire space. Simultaneously it is not true that $\mathcal{M}\left(\mathcal{T}_{\Psi}\right)=\mathcal{N}$ nor $\mathcal{B}\left(\mathcal{T}_{\Psi}\right) \subset \mathcal{L}$. So, assumption in condition $d$ ) is necessary. There is an example (in [27]) of a set $A \in \mathcal{L}$ such that $\operatorname{int}_{\mathcal{T}_{\Psi}}(A) \neq A \cap \Phi_{\Psi}(A)$, so inclusion in $e$ ) can be proper. 
Remark 25.29. If $X \neq \emptyset, \mathcal{S}=2^{X}, \mathcal{J}=\{\emptyset\}$, then the operator $\Phi(A)=A$ is the almost lower density operator on $(X, \mathcal{S}, \mathcal{J})$ such that $\mathcal{T}_{\Phi}$ is the discrete topology. Then it is clear that inverse of $b)$ is not satisfied.

Remark 25.30. Let us consider a lower density operator $\Phi$ on $(\mathbb{R}, \mathcal{L}, \mathcal{N})$ given by formula:

$$
\forall_{A \in \mathcal{L}} \quad \Phi(A)=\left\{\begin{array}{cl}
\mathbb{R}, & \text { if } \mathbb{R} \backslash A \in \mathcal{N}, \\
\emptyset, & \text { if } \mathbb{R} \backslash A \notin \mathcal{N} .
\end{array}\right.
$$

Then

$$
\mathcal{T}_{\Phi}=\{A \subset \mathbb{R}: A=\emptyset \vee \mathbb{R} \backslash A \in \mathcal{N}\}
$$

is a topology generated by $\Phi$ such that $\mathcal{M}\left(\mathcal{T}_{\Phi}\right)=\mathcal{N}$. Evidently $\mathcal{B}\left(\mathcal{T}_{\Phi}\right) \subset \mathcal{L}$ and the inverse inclusion is not true. Hence inclusion in $d$ ) can be proper.

Theorem 25.31. If $\Phi_{1}, \Phi_{2}$ are equivalent almost lower density operators on $(X, \mathcal{S}, \mathcal{J})$ and $\mathcal{T}_{\Phi_{1}}, \mathcal{T}_{\Phi_{2}}$ topologies on $X$ generated by $\Phi_{1}, \Phi_{2}$ respectively, then

$1^{\circ} \mathcal{M}\left(\mathcal{T}_{\Phi_{1}}\right)=\mathcal{M}\left(\mathcal{T}_{\Phi_{2}}\right)$;

$2^{\circ} \mathcal{B}\left(\mathcal{T}_{\Phi_{1}}\right)=\mathcal{B}\left(\mathcal{T}_{\Phi_{2}}\right)$.

First we need the following lemma.

Lemma 25.32. If $\Phi_{1}, \Phi_{2}$ are equivalent almost lower density operators on $(X, \mathcal{S}, \mathcal{J})$, then for every set $A \in \mathcal{T}_{\Phi_{1}}$ there exists a set $E \in \mathcal{J}$ such that $A \backslash E \in \mathcal{T}_{\Phi_{2}}$.

Proof. Let $A \in \mathcal{T}_{\Phi_{1}}$, hence $A \subset \Phi_{1}(A)$. Put $E=\Phi_{1}(A) \triangle \Phi_{2}(A)$. Then $E \in \mathcal{J}$ and $A \subset \Phi_{2}(A) \triangle E$. Thus $A \subset \Phi_{2}(A) \cup E$. This implies that $A \backslash E \subset \Phi_{2}(A)=\Phi_{2}(A \backslash E)$. Therefore $A \backslash E \in \mathcal{T}_{\Phi_{2}}$.

Proof of Theorem 25.31. First we show that the families of nowhere dense sets with respect to topologies $\mathcal{T}_{\Phi_{1}}$ and $\mathcal{T}_{\Phi_{2}}$ are equal. Let $A$ be a nowhere dense set with respect to $\mathcal{T}_{\Phi_{1}}$ and $V_{2}$ nonempty $\mathcal{T}_{\Phi_{2}}$-open set. By Lemma 25.32 there exists a set $E_{1} \in \mathcal{J}$ such that $V_{2} \backslash E_{1} \in \mathcal{T}_{\Phi_{1}}$. Moreover, there exists a nonempty set $V_{1} \in \mathcal{T}_{\Phi_{1}}$ such that $V_{1} \subset V_{2} \backslash E_{1}$ and $A \cap V_{1}=\emptyset$. By Lemma 25.32 there exists a set $E_{2} \in \mathcal{J}$ such that $V_{1} \backslash E_{2} \in \mathcal{T}_{\Phi_{2}}$. Obviously $V_{1} \backslash E_{2} \neq \emptyset$. Thus $V_{1} \backslash E_{2} \subset V_{2}$ and $A \cap\left(V_{1} \backslash E_{2}\right)=\emptyset$. Hence $A$ is a nowhere dense set with respect to $\mathcal{T}_{\Phi_{2}}$. In a similar way we prove the inverse inclusion.

Condition $1^{\circ}$ is now an immediate consequence of the equality of the families of nowhere dense sets with respect to topologies $\mathcal{T}_{\Phi_{1}}$ and $\mathcal{T}_{\Phi_{2}}$.

Now we prove that $\mathcal{B}\left(\mathcal{T}_{\Phi_{1}}\right)=\mathcal{B}\left(\mathcal{T}_{\Phi_{2}}\right)$. Suppose that $A \in \mathcal{B}\left(\mathcal{T}_{\Phi_{1}}\right)$. Then $A=V \triangle Y$, where $V \in \mathcal{T}_{\Phi_{1}}$ and $Y \in \mathcal{M}\left(\mathcal{T}_{\Phi_{1}}\right)$. By Lemma 25.32 there exists a set $E_{1} \in \mathcal{J}$ such that $V \backslash E_{1} \in \mathcal{T}_{\Phi_{2}}$. Hence $A=\left[\left(V \backslash E_{1}\right) \cup\left(V \cap E_{1}\right)\right] \triangle Y$. 
Since $V \backslash E_{1} \in \mathcal{T}_{\Phi_{2}}$ and $\left(V \cap E_{1}\right) \in \mathcal{J} \subset \mathcal{M}\left(\mathcal{T}_{\Phi_{2}}\right)$ and $Y \in \mathcal{M}\left(\mathcal{T}_{\Phi_{2}}\right)$, we have $A \in \mathcal{B}\left(\mathcal{T}_{\Phi_{2}}\right)$. In a similar way we prove that $\mathcal{B}\left(\mathcal{T}_{\Phi_{2}}\right) \subset \mathcal{B}\left(\mathcal{T}_{\Phi_{1}}\right)$.

In the further consideration we apply the following proposition.

Proposition 25.33 (cf. 1.14 in [10]). Let $\Phi$ be the almost lower density operators on $(X, \mathcal{S}, \mathcal{J})$. Then the following conditions are equivalent:

1. $\forall_{A \in \mathcal{S}} \quad A \backslash \Phi(A) \in \mathcal{J}$;

2. $\forall A \in \mathcal{S} \quad A \triangle \Phi(A) \in \mathcal{J}$.

Theorem 25.34. If $\Phi$ is an almost lower density operator on $(X, \mathcal{S}, \mathcal{J})$ generating topology $\mathcal{T}_{\Phi}$, then

(i) $\mathcal{M}\left(\mathcal{T}_{\Phi}\right)=\mathcal{J}$ if and only if there exists a $\sigma$-algebra $\mathcal{S}^{\prime} \subset \mathcal{S}$ such that $\Phi^{\prime}=\Phi_{\mid \mathcal{S}^{\prime}}$ is a lower density operator on $\left(X, \mathcal{S}^{\prime}, \mathcal{J}\right)$ and $\mathcal{T}_{\Phi}=\mathcal{T}_{\Phi^{\prime}} ;$

(ii) $\mathcal{M}\left(\mathcal{T}_{\Phi}\right)=\mathcal{J}$ and $\mathcal{B}\left(\mathcal{T}_{\Phi}\right)=\mathcal{S}$ if and only if $\Phi$ is a lower density operator on $(X, \mathcal{S}, \mathcal{J})$.

Proof. (i) Necessity. Let $\mathcal{S}^{\prime}=\mathcal{B}\left(\mathcal{T}_{\Phi}\right)$. Then $\mathcal{J} \subset \mathcal{S}^{\prime} \subset \mathcal{S}$. It is sufficient to prove that $\Phi^{\prime}=\Phi_{\mid \mathcal{S}^{\prime}}$ is a lower density operator on $\left(X, \mathcal{S}^{\prime}, \mathcal{J}\right)$. For every $A \in \mathcal{S}^{\prime}$ we have $A=B \triangle C$, where $B \in \mathcal{T}_{\Phi}, C \in \mathcal{J}$. Hence $\Phi^{\prime}(A)=\Phi(A)=\Phi(B) \supset$ $B$. It follows that $A \backslash \Phi^{\prime}(A) \subset A \backslash B \in \mathcal{J}$. By Proposition 25.33 we conclude that $\Phi^{\prime}(A) \backslash A \in \mathcal{J}$. So $\Phi^{\prime}(A) \triangle A \in \mathcal{J}$ and $\Phi^{\prime}$ is a lower density operator on $\left(X, \mathcal{S}^{\prime}, \mathcal{J}\right)$. The equality $\mathcal{T}_{\Phi}=\mathcal{T}_{\Phi^{\prime}}$ is obvious.

Sufficiency. If $\Phi^{\prime}$ is a lower density operator on $\left(X, \mathcal{S}^{\prime}, \mathcal{J}\right)$ generating topology $\mathcal{T}_{\Phi^{\prime}}$, then by Theorem $25.3, \mathcal{M}\left(\mathcal{T}_{\Phi^{\prime}}\right)=\mathcal{J}$. Since $\mathcal{M}\left(\mathcal{T}_{\Phi}\right)=\mathcal{M}\left(\mathcal{T}_{\Phi^{\prime}}\right)$, we get $\mathcal{J}=\mathcal{M}\left(\mathcal{T}_{\Phi}\right)$.

(ii) Necessity. If $\mathcal{M}\left(\mathcal{T}_{\Phi}\right)=\mathcal{J}$, then $\Phi_{\mid \mathcal{S}^{\prime}}$, where $\mathcal{S}^{\prime}=\mathcal{B}\left(\mathcal{T}_{\Phi}\right)$, is a lower density operator on $\left(X, \mathcal{S}^{\prime}, \mathcal{J}\right)$. Since $\mathcal{S}=\mathcal{S}^{\prime}$, we have $\Phi$ is a lower density operator on $(X, \mathcal{S}, \mathcal{J})$.

Sufficiency. If $\Phi$ is a lower density operator on $(X, \mathcal{S}, \mathcal{J})$, then by Theorem 25.3 we obtain $\mathcal{M}\left(\mathcal{T}_{\Phi}\right)=\mathcal{J}$ and $\mathcal{B}\left(\mathcal{T}_{\Phi}\right)=\mathcal{S}$.

Example 25.35. Let $Y \subset(0,1)$ and $Y \notin \mathcal{L}$. If we put

$$
\forall A \in \mathcal{L} \quad \Phi(A)=\left\{\begin{array}{cl}
\mathbb{R}, & \text { if } \mathbb{R} \backslash A \in \mathcal{N}, \\
\Phi_{d}(A) \cap Y, & \text { if } \mathbb{R} \backslash A \notin \mathcal{N},
\end{array}\right.
$$

then we obtain an almost lower density operator on $(\mathbb{R}, \mathcal{L}, \mathcal{N})$ generating topology

$$
\mathcal{T}_{\Phi}=\{A \subset \mathbb{R}: \mathbb{R} \backslash A \in \mathcal{N}\} \cup\left(\mathcal{T}_{d} \cap 2^{Y}\right)
$$

but $\Phi((0,1))=\Phi_{d}((0,1)) \cap Y \notin \mathcal{L}$. 
In the context of the above observation we see the range of an almost density operator can be wider then the $\sigma$-algebra $\mathcal{S}$. However we have the following theorem.

Theorem 25.36. If $\Phi$ is an almost lower density operator on $(X, \mathcal{S}, \mathcal{J})$, then there exists a subfamily $\mathcal{R} \subset \mathcal{S}$ such that $\mathcal{J} \subset \mathcal{R}, X \in \mathcal{R}, \mathcal{R}$ is closed under finite intersections and an almost lower density operator $\Phi^{\prime}: \mathcal{R} \rightarrow \mathcal{S}$ on $(X, \mathcal{R}, \mathcal{J})$ such that

$$
\mathcal{T}_{\Phi^{\prime}}=\left\{A \in \mathcal{R}: A \subset \Phi^{\prime}(A)\right\}=\mathcal{T}_{\Phi} .
$$

Proof. Let $\mathcal{R}=\{A \in \mathcal{S}: \Phi(A) \in \mathcal{S}\}$. Then $\emptyset, X \in \mathcal{R}, \mathcal{J} \subset \mathcal{R}$ and $\mathcal{R}$ is closed under finite intersections. Let $\Phi^{\prime}=\Phi_{\mid \mathcal{R}}$ be the restriction of $\Phi$ to the family $\mathcal{R}$. It is clear that $\Phi^{\prime}: \mathcal{R} \rightarrow \mathcal{S}$ is an almost lower density operator on $(X, \mathcal{R}, \mathcal{J})$. We show that $\mathcal{T}_{\Phi^{\prime}}=\mathcal{T}_{\Phi}$. It is sufficient to show that $\mathcal{T}_{\Phi} \subset \mathcal{T}_{\Phi^{\prime}}$. If $A \in \mathcal{T}_{\Phi}$, then $A \in \mathcal{S}$ and $A \subset \Phi(A)$. Since $\Phi(A) \backslash A \in \mathcal{J}$, we have $\Phi(A)=A \cup(\Phi(A) \backslash A) \in \mathcal{S}$ and $A \in \mathcal{R}$. From the inclusion $A \subset \Phi^{\prime}(A)$ we obtain $A \in \mathcal{T}_{\Phi^{\prime}}$.

Theorem 25.37 (cf. [11]). Let $\langle X, \tau\rangle$ be a topological space such that $X \notin \mathcal{M}(\tau)$ and $\Phi$ be an almost lower density operator on $(X, \mathcal{B}(\tau), \mathcal{M}(\tau))$. Then $\Phi$ generates topology $\mathcal{T}_{\Phi}$. Moreover, if there exists a $\tau$-dense set $D \in \mathcal{M}(\tau)$ and $\tau \subset \mathcal{T}_{\Phi}$, then the topological space $\left\langle X, \mathcal{T}_{\Phi}\right\rangle$ is not regular.

Proof. Since the pair $(\mathcal{B}(\tau), \mathcal{M}(\tau))$ has the hull property, then by Theorem 25.23

$$
\mathcal{T}_{\Phi}=\{A \in \mathcal{B}(\tau): A \subset \Phi(A)\}
$$

is a topology on $X$.

Let $D \in \mathcal{M}(\tau)$ be a $\tau$-dense set and $\tau \subset \mathcal{T}_{\Phi}$. First we prove that if $D \subset W \in$ $\mathcal{T}_{\Phi}$, then $X \backslash W \in \mathcal{M}(\tau)$. Evidently $W=B \triangle C$, where $B \in \tau, C \in \mathcal{M}(\tau)$. We show that $B$ is $\tau$-dense. Suppose that there exists a nonempty set $E \in \tau$ such that $B \cap E=\emptyset$. By the assumption that $\tau \subset \mathcal{T}_{\Phi}$ we have $W \cap E \in \mathcal{T}_{\Phi}$ and thus

$$
W \cap E \subset \Phi(W \cap E)=\Phi(B \cap E)=\emptyset .
$$

Hence $W \cap E=\emptyset$ and it contradicts the fact that $W$ is $\tau$-dense. Thus $B$ is $\tau$-dense and $\tau$-open. Therefore $X \backslash B \in \mathcal{M}(\tau)$ and $X \backslash W \in \mathcal{M}(\tau)$.

Taking a $\mathcal{T}_{\Phi}$-closed set $D$ and a point $x_{0} \in X \backslash D$ we see that for $\mathcal{T}_{\Phi}$-open and disjoint sets $W$ and $V$ such that $D \subset W$ and $x_{0} \in V$ we get $X \backslash W \in \mathcal{M}(\tau)$ and $V \notin \mathcal{M}(\tau)$. So $W \cap V \neq \emptyset$. This contradiction forces us to conclude that the topological space $\left\langle X, \mathcal{T}_{\Phi}\right\rangle$ is not regular.

Corollary 25.38. If $\Phi$ is an almost lower density operator on $(\mathbb{R}, \mathcal{B}$ or, $\mathcal{M})$ such that $\mathcal{T}_{\text {nat }} \subset \mathcal{T}_{\Phi}$, then the topological space $\left\langle\mathbb{R}, \mathcal{T}_{\Phi}\right\rangle$ is not regular. 
Now we will concentrate on the topologies generated by almost lower density operators invariant with respect to translation or multiplication.

Definition 25.39. We shall say that a topology $\tau$ on $\mathbb{R}$ is invariant with respect to translation (multiplication) if

$$
\forall_{A \in \tau} \quad \forall_{t \in \mathbb{R}} \quad A+t \in \tau \quad\left(\forall_{A \in \tau} \quad \forall_{\alpha \in \mathbb{R} \backslash\{0\}} \quad \alpha A \in \tau\right) .
$$

Definition 25.40. We shall say that a measurable space $(\mathbb{R}, \mathcal{S}, \mathcal{J})$ is invariant with respect to translation (multiplication) if
i) $\forall_{A \in \mathcal{S}} \quad \forall_{t \in \mathbb{R}} \quad A+t \in \mathcal{S} \quad\left(\forall_{A \in \mathcal{S}} \quad \forall_{\alpha \in \mathbb{R} \backslash\{0\}} \quad \alpha A \in \mathcal{S}\right)$,
ii) $\forall_{A \in \mathcal{J}} \quad \forall_{t \in \mathbb{R}} \quad A+t \in \mathcal{J} \quad\left(\forall_{A \in \mathcal{J}} \quad \forall_{\alpha \in \mathbb{R} \backslash\{0\}} \quad \alpha A \in \mathcal{J}\right)$,

Definition 25.41. We shall say that an almost lower density operator $\Phi$ on an invariant with respect to translation (multiplication) measurable space $(\mathbb{R}, \mathcal{S}, \mathcal{J})$ is invariant with respect to translation (multiplication) if

$$
\forall_{A \in \mathcal{S}} \quad \forall_{t \in \mathbb{R}} \quad \Phi(A+t)=\Phi(A)+t \quad\left(\forall_{A \in \mathcal{S}} \quad \forall_{\alpha \in \mathbb{R} \backslash\{0\}} \quad \Phi(\alpha A)=\alpha \Phi(A)\right) .
$$

Evidently, we have the following property.

Property 25.42. If $\Phi$ is an almost lower density operator invariant with respect to translation (multiplication) on a measurable space $(\mathbb{R}, \mathcal{S}, \mathcal{J})$ invariant with respect to translation (multiplication) generating topology $\mathcal{T}_{\Phi}$, then $\mathcal{T}_{\Phi}$ is invariant with respect to translation (multiplication).

Property 25.43. Let $(\mathbb{R}, \mathcal{S}, \mathcal{J})$ be a measurable space invariant with respect to translation and multiplication. If $\Phi$ is an almost lower density operator on $(\mathbb{R}, \mathcal{S}, \mathcal{J})$ invariant with respect to translation generating topology $\mathcal{T}_{\Phi}$, then $\mathcal{T}_{\Phi}$ is invariant with respect to translation and, moreover, $\mathcal{T}_{\Phi}$ is invariant with respect to multiplication if and only if

$$
\forall_{A \in \mathcal{T}_{\Phi}} \quad \forall_{\alpha \in \mathbb{R} \backslash\{0\}} \quad(0 \in A \Longrightarrow 0 \in \Phi(\alpha A)) .
$$

Proof. By the previous property $\mathcal{T}_{\Phi}$ is invariant with respect to translation. Let $\alpha \in \mathbb{R} \backslash\{0\}$ and $A \in \mathcal{T}_{\Phi}$. We show that $\alpha A \in \mathcal{T}_{\Phi}$. Let $y \in \alpha A$, then $y / \alpha \in$ $A \subset \Phi(A)$. Hence $0 \in A-y / \alpha$. By assumption $0 \in \Phi(\alpha A-y)=\Phi(\alpha A)-y$. Therefore $y \in \Phi(\alpha A)$ and it follows that $\alpha A \subset \Phi(\alpha A)$. Since $\alpha A \in \mathcal{S}$, we obtain $\alpha A \in \mathcal{T}_{\Phi}$.

Now assume that $\mathcal{T}_{\Phi}$ is invariant with respect to multiplication. Suppose that

$$
\exists_{A \in \mathcal{T}_{\Phi}} \quad \exists_{\alpha \in \mathbb{R} \backslash\{0\}} \quad(0 \in A \wedge 0 \notin \Phi(\alpha A)) .
$$


Since $\alpha A \in \mathcal{T}_{\Phi}$, then $\alpha A \subset \Phi(\alpha A)$. Moreover, $0 \in \Phi(\alpha A)$ because $0 \in \alpha A$. This contradiction completes the proof.

\section{References}

[1] V. Aversa, W. Wilczyński, Simple density topology, Rend. Circ. Mat. Palermo, serie II, 53(3) (2004), 344-352.

[2] K. Ciesielski, L. Larson, K. Ostaszewski, I-Density Continuous Functions, Mem. Amer. Math. Soc. 107(515), 1994.

[3] M. Filipczak, T. Filipczak, On f-density topologies, Topology Appl. 155 (2008), 19801989.

[4] M. Filipczak, J. Hejduk, On topologies associated with the Lebesgue measure, Tatra Mt. Math. Publ. 28 (2004), 187-197.

[5] C. Goffman, C. J. Neugebauer, T. Nishiura, Density topology and approximate continuity, Duke Math. J. 28 (1961), 497-505.

[6] C. Goffman, D. Waterman, Approximately continuous transformations, Proc. Amer. Math. Soc. 12 (1961), 116-121.

[7] T. R. Hamlett, D. Janković, D. A. Rose, Lower density topologies, Ann. New York Acad. Sci., Papers on General Topology and Applications 704 (1993), 309-321.

[8] O. Haupt, C. Pauc, La topologie de Denjoy approximative envisagée comme vraie topologie, C. R. Acad. Sci. Paris 234 (1952), 390-392.

[9] J. Hejduk, On density topologies generated by functions, Tatra Mt. Math. Publ. 40 (2008), 133-141.

[10] J. Hejduk, On density topologies with respect to invariant $\sigma$-ideals, J. Appl. Anal. 8(2) (2002), 201-219.

[11] J. Hejduk, On the regularity of topologies in the family of sets having the Baire property, Filomat (submitted).

[12] J. Hejduk, G. Horbaczewska, On $\mathcal{I}$-density topologies with respect to a fixed sequence, Reports on Real Analysis (Rowy), (2003), 78-85.

[13] J. Hejduk, R. Wiertelak, On the generalization of the density topology on the real line, Mathematica Slovaca (to appear).

[14] E. W. Hobson, The theory of functions of a real variable and the theory of Fourier's series, vol. I. Dover Publications, Inc., New York, 1958.

[15] G. Horbaczewska, The family of $\mathcal{I}$-density type topologies, Comment. Math. Univ. Carolinae 46(4) (2005), 735-745.

[16] J. M. Jȩdrzejewski, On limit numbers of real functions, Fund. Math. 83(3) (1973/1974), 269-281.

[17] K. Kuratowski, Topology, vol 1, Polish Scientific Publications, Warsaw, 1966.

[18] J. Lukeš, J. Malý, L. Zajiček, Fine Topology Methods in Real Analysis and Potential Theory, Lecture Notes in Math. 1189, Springer-Verlag, Berlin 1986.

[19] E. Łazarow, A. Vizvary, $\Psi_{\mathcal{I}}$-density topology, Pr. Nauk Akad. Jana Długosza Czȩst. Mat. 15 (2010), 67-80.

[20] D. Maharam, On a theorem of von Neumann, Proc. Amer. Math. Soc. 9 (1958), 987-994.

[21] J. C. Oxtoby, Measure and category, Springer-Verlag, Berlin 1980. 
[22] W. Poreda, E. Wagner-Bojakowska, W. Wilczyński, A category analogue of the density topology, Fund. Math. 125(2) (1985), 167-173.

[23] W. Poreda, W. Wilczyński, Topology similar to the density topology, Bull. Soc. Sci. Lett. Łódź, 34 (2001), 55-60.

[24] F. D. Tall, The density topology, Pacific. J. Math. 62(1) (1976), 275-284.

[25] M. Terepeta, E. Wagner-Bojakowska, $\psi$-density topology, Rend. Circ. Mat. Palermo, Serie II 48(3) (1999), 451-476.

[26] E. Wagner-Bojakowska, Remarks on $\psi$-density topology, Atti Sem. Mat. Fis. Univ. Modena 49(1) (2001), 79-87.

[27] E. Wagner-Bojakowska, W. Wilczyński, The union of $\psi$-density topologies, Atti Sem. Mat. Fis. Univ. Modena 50(2) (2002), 313-326.

[28] R. Wiertelak, A generalization of density topology with respect to category, Real Anal. Exchange 32(1) (2006/2007), 273-286.

[29] W. Wilczyński, A category analogue of the density topology, approximate continuity and the approximate derivative, Real Anal. Exchange 10(2) (1984-85), 241-265.

[30] W. Wilczyński, Density topologies, Chapter 15 in Handbook of Measure Theory, Ed. E. Pap. Elsevier (2002), 675-702.

[31] W. Wilczyński, W. Wojdowski, A category $\psi$-density topology, Cent. Eur. J. Math. 9(5) (2011), 1057-1066.

[32] W. Wilczyński, W. Wojdowski, Complete density topology, Indag. Math., New series, 18(2) (2007), 295-303.

[33] W. Wojdowski, Density topologies involving measure and category, Demonstratio Math. 22(3) (1989), 797-812.

\section{JACEK HEJDUK}

Faculty of Mathematics and Computer Science, Łódź University

ul. Banacha 22, 90-238 Łódź, Poland

E-mail: hejduk@math.uni.lodz.pl

\section{RENATA WIERTELAK}

Faculty of Mathematics and Computer Science, Łódź University

ul. Banacha 22, 90-238 Łódź, Poland

E-mail: wiertelak@math.uni.lodz.pl 